\title{
Pathogenesis analysis of pituitary adenoma based on gene expression profiling
}

\author{
WEIMIN WANG ${ }^{1,2}$, ZHIMING XU ${ }^{2}$, LI FU ${ }^{3}$, WEI LIU ${ }^{2}$ and XINGANG LI ${ }^{1,4}$ \\ ${ }^{1}$ School of Medicine, Shandong University, Jinan, Shandong 250012; Departments of ${ }^{2}$ Neurosurgery and ${ }^{3}$ General Surgery, \\ Qingdao Municipal Hospital, Qingdao, Shandong 266071; ${ }^{4}$ Department of Neurosurgery, \\ Qilu Hospital of Shandong University, Jinan, Shandong 250012, P.R. China
}

Received February 13, 2014; Accepted September 8, 2014

DOI: $10.3892 / \mathrm{ol} .2014 .2613$

\begin{abstract}
The aim of the current study was to investigate the pathogenesis of pituitary adenoma through screening of the differentially-expressed genes (DEGs) and proteins in normal pituitary and pituitary adenoma tissues, and analyzing the interactions among them. Following the acquisition of gene expression profiling data from a public functional genomics data repository, Gene Expression Omnibus, DEGs were screened in normal pituitary and pituitary adenoma tissues. Upregulated and downregulated DEGs were further identified through gene ontology functional enrichment analysis. Subsequently, the DEGs were mapped to the Search Tool for the Retrieval of Interacting Genes database, and the protein-protein interaction (PPI) networks of the upregulated and downregulated DEGs were constructed. Finally, the functional modules of the PPI network of the downregulated DEGs were analyzed. In total, 211 upregulated and 413 downregulated DEGs were screened between the normal pituitary and pituitary adenoma samples. Downregulated DEGs were associated with certain functions, including the immune response, hormone regulation and cell proliferation. Upregulated genes were associated with cation transport functions. Five modules were acquired from the PPI network of the downregulated DEGs. Transcription factors, including signal transducer and activator of transcription 3 (STAT3), interleukin 6 (IL-6), B-cell lymphoma 6 protein, early growth response $1, \mathrm{POU} 1 \mathrm{~F} 1$, jun B proto-oncogene and FOS were the core nodes in the functional modules. In summary, the DEGs and proteins were identified through screening gene expression profiling and PPI networks. The results of the present study indicated that low expression levels of hormone- and immune-related genes facilitated
\end{abstract}

Correspondence to: Dr Xingang Li, School of Medicine, Shandong University, 44 Wenhua Xi Road, Jinan, Shandong 250012, P.R. China

E-mail: xinggll23@hotmail.com

Key words: pituitary adenoma, differentially-expressed genes, interleukin 6, signal transducer and activator of transcription 3, POU1F1 the occurrence of pituitary adenoma. Low expression levels of IL-6 and STAT3 were significant in the dysimmunity of pituitary adenoma. Furthermore, the low expression level of POU1F1 contributed to the reduction in pituitary hormone secretion.

\section{Introduction}

The pituitary gland, as an important endocrine organ, performs vital roles in organismal modulation through secreting several key hormones, including prolactin (PRL), growth hormone $(\mathrm{GH})$, adrenocorticotropic hormone and thyroid-stimulating hormone (TSH) $(1,2)$. By regulating the hormone secretion of a target gland, the anterior pituitary participates in the development and normalization of tissues (3). The abnormality of the pituitary may severely disrupt the metabolic homeostasis, damaging various organs to different extents (4). Pituitary adenoma is a distinctive intracranial tumor developing in the anterior pituitary gland, which exhibits the characteristics of a tumor and an endocrine disorder. Pituitary adenoma, which is caused by mutations in a series of significant pituitary genes, including protein kinase $\mathrm{C}$, p16, and growth arrest and DNA damage-45G (GADD45G), has provoked continuous attention among endocrinologists due to its variability in clinical presentation and the unpredictability of tumor growth (5).

As demonstrated in previous studies, pituitary adenoma may affect human development in a negative manner from a variety of perspectives. Excess hormone secretion by the pituitary gland, caused by pituitary adenoma, produces several metabolic disorders and visceral injuries (6). By contrast, other hormones are downregulated due to the compression of the tumor, which subsequently results in the functional decline of the target glands (7).

To date, a wealth of studies have been conducted on the surgical treatment and chemotherapy of pituitary adenoma $(8,9)$. Studies have shown that the gene and protein expression of certain regulatory factors have vital roles in pituitary adenoma. Vogelstein et al (10) found that p53 inhibits the development of a pituitary adenoma and this function may be restrained by pleomorphic adenoma gene-like 1 in combination with reprimo, TP53-dependent G2 arrest mediator candidate, p21 and phorbol-12-myristate-13-acetate-induced protein 1. It is well acknowledged that the overexpression 
of GADD45 $\beta$ may inhibit tumor growth through activating apoptosis-inhibiting factors, which indicates that GADD $45 \beta$ may also serve a potential inhibitor of pituitary adenoma (11). Pituitary adenoma may be associated with an increase or decrease in a variety of gene expression levels; the majority of these changes also exert regulatory effects on tumorigenesis. Although a number of studies have reported controversial effects of pituitary adenoma on the potential target genes, no effective detection method is available using the flux way to systematically detect the gene and protein differential expression caused by pituitary adenoma (12-14).

The present study aimed to investigate the types and changes of gene expression in pituitary adenoma compared with normal pituitary tissues through gene expression profiling. Subsequently, by establishing a protein-protein interaction (PPI) network of differentially-expressed genes (DEGs), the effects of differential proteins on pituitary adenoma, and the interactions among diverse differential proteins were analyzed.

\section{Materials and methods}

Data preprocessing and acquisition of gene expression profiling. The gene expression profile GSE26966(11) was downloaded from the public functional genomics data repository, Gene Expression Omnibus (http://www.ncbi.nlm.nih.gov/geo/) database. Among the total 23 samples that were investigated, nine samples were from normal pituitary tissues and 14 samples were pituitary adenoma tissues. The annotation information for all probe sets was provided by Affymetrix Human Genome U133 Plus 2.0 Array (Affymetrix, Inc., Santa Clara, CA, USA). For data processing and differential expression analysis, the probe-level data was converted from the CEL file format into the expression values of a probe matrix by robust multi-array average (RMA) in Affy package (15), and the serial numbers were transferred into gene names by platform R/Bioconductor note package (16). Finally, as one gene has numerous corresponding probes, the average value of all expression value of probes was calculated as the expression value of a single gene.

$D E G$ screening. The Bayesian linear model of the limma package in the R software (5) was used to identify DEGs in pituitary adenoma tissues compared with those in the normal pituitary gland. Only genes with a log fold-change value of $>1.5$ and a false discovery rate (FDR) of $<0.05$ were selected as DEGs. To ensure that the screened DEGs could well characterize the samples, clustering analysis and dendrograms for DEGs were established.

Functional enrichment analysis of DEGs. Gene ontology (GO) functional enrichment analysis was conducted using the Database for Annotation Visualization and Integrated Discovery (DAVID) online tools (17) to study the functions of upregulated and downregulated DEGs, respectively. The cut-off criteria were the FDR and Bonferroni correction (18); $\mathrm{P}<0.05$ was considered to indicate a statistically significant difference.

PPI network construction. The PPI pairs were acquired by directly mapping the DEGs to the Search Tool for the Retrieval of Interacting Genes (STRING) database (19); the PPI network was constructed by PPI pairs whose protein interaction scores were $>0.7$. The protein interaction scores were calculated using the following formula (20):

$$
\begin{aligned}
S(\mathrm{e}(x, y)) & =f(\operatorname{diff}(x), \operatorname{corr}(x, y), \operatorname{diff}(y)) \\
& =-2 \sum_{i=1}^{k} \log _{-}\left(p_{i}\right)
\end{aligned}
$$

$\operatorname{Diff}(\mathrm{x})$ and $\operatorname{diff}(\mathrm{y})$ represent differential expression assessments of genes $\mathrm{x}$ and $\mathrm{y}$, respectively. Corr $(\mathrm{x}, \mathrm{y})$ represents their correlation between genes $x$ and $y$. Where $k=3, p_{1}$ and $\mathrm{p}_{2}$ are the P-values of differential expression of two nodes, $\mathrm{p}_{3}$ is the P-value (20) of their coexpression.

Specific transcription factors in the PPI network of pituitary adenoma were excavated using the TRANSFAC database (21).

Analysis of PPI modules. Modules in the PPI network of downregulated DEGs were selected using the cluster ONE package in the Cytoscape software (22). Modules with P-values of network minimum density $>0.05$ and node numbers $>10$ were screened. Sequentially, GO analysis of these modules was conducted using DAVID.

\section{Results}

DEGs in pituitary adenomas. Following the pretreatment of dataset GSE26966 and DEG screening, a total of 624 DEGs, including 211 upregulated genes and 413 downregulated genes, between pituitary adenoma and normal pituitary tissue samples were acquired. A clustering diagram of DEGs is shown in Fig. 1.

Functional enrichment analysis of DEGs. Significant enrichment GO items of upregulated and downregulated DEGs are shown in Table I. The functions of the downregulated genes in the pituitary adenoma were predominantly associated with the immune response, hormone regulation and cell proliferation. The upregulated genes were associated with cation transport, such as metal ion transport. The stimulation and adjustment of downregulated DEGs on hormones may be associated with hormone secretion in the pituitary gland.

PPI network construction. The PPI network was constructed by PPI pairs based on the STRING database. The PPI network of downregulated genes was composed of 158 nodes and 241 lines (Fig. 2), and the network of upregulated genes consisted of 16 nodes and 10 lines (Fig. 3). In total, 13 transcription factors were included in the PPI network of the downregulated genes, such as early growth response 1 (EGR1), signal transducer and activator of transcription (STAT3), jun B proto-oncogene (JUNB) and FOS, while only the transcription factor GATA-binding protein-3 was included in the PPI network of upregulated genes.

Functional modules of the PPI network of downregulated DEGs. The PPI network of the downregulated DEGs in pituitary adenoma exhibited centralization, and certain node proteins of the network, including EGR1, STAT3, JUNB and FOS, were the common transcription factors in cancer (23); the PPI network of upregulated DEGs was sparsely populated. Through the comparison between these two PPI networks 


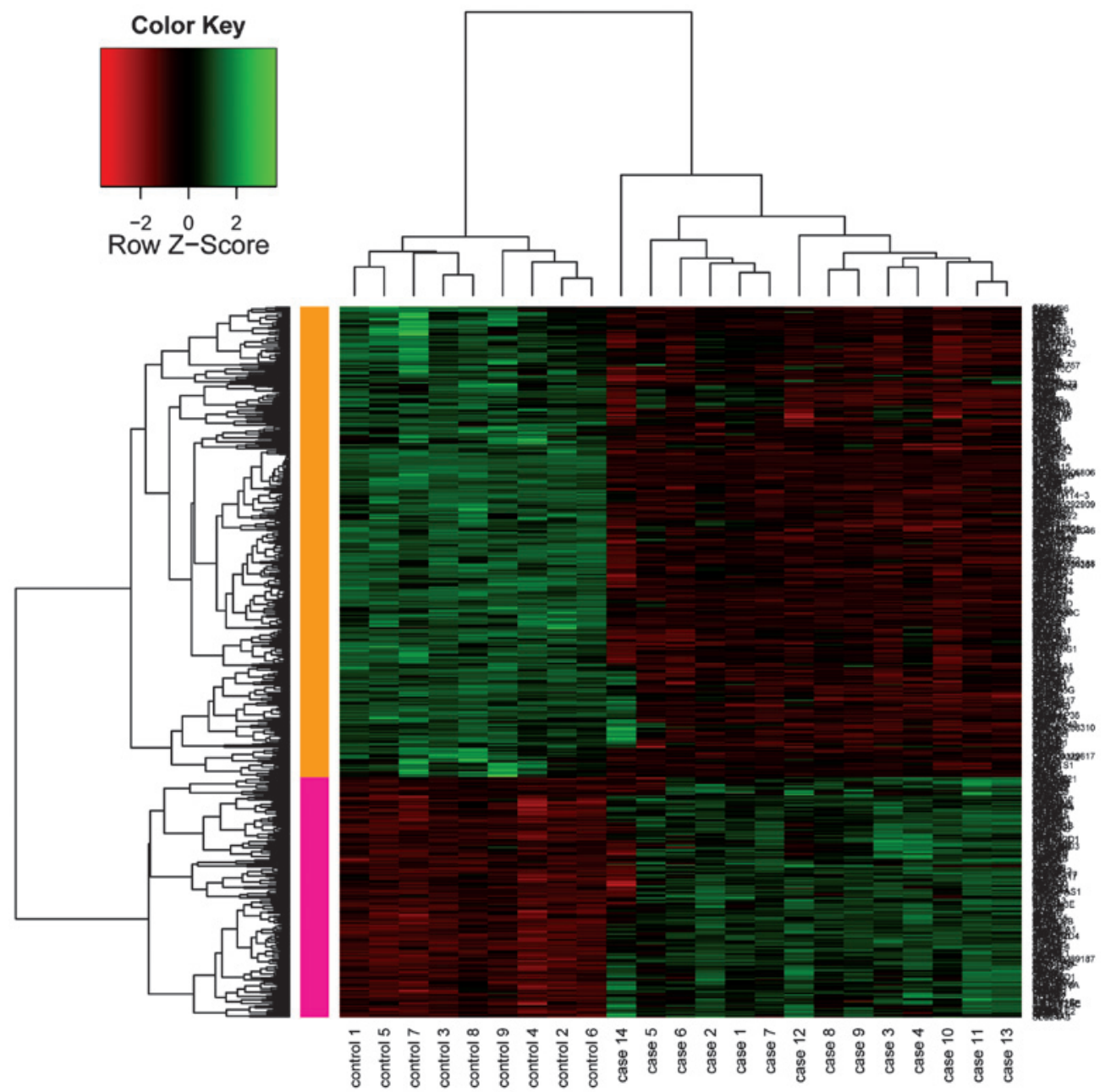

Figure 1. Clustering diagram of DEGs. The bottom abscissa shows names of specimens (control indicates samples of normal pituitary gland, and case indicates samples of pituitary adenoma). The top abscissa indicates clustering of specimens. The right longitudinal axis shows DEGs, while the left longitudinal axis indicates the clustering of DEGs. Red represents downregulated genes and green represents upregulated genes. Two major groups were identified in the clustering diagram of specimens: DEGs and samples of pituitary adenoma. The clustering of DEGs may also be divided into two subgroups: Downregulated and upregulated genes of pituitary adenoma samples. DEG, differentially-expressed genes.

(Figs. 2 and 3), downregulated genes were observed to be significant in pituitary adenoma. Therefore, the current study focused on the analysis of functional modules in the PPI network of downregulated DEGs.

Five modules were acquired (Fig. 4), with module one exhibiting the largest number of nodes $(n=32)$. In addition to module one, the functions of the remaining four modules were significantly enriched (Table II). In module one, interleukin-6 (IL-6), IL-6-receptor (IL6R) and STAT3 were observed to be enriched in the IL-6-mediated signaling pathway $(\mathrm{P}<0.05)$, and IL-6, angiotensinogen, leukemia inhibitory factor, haptoglobin, B-cell lymphoma 6 protein (BCL6), peroxisome proliferator-activated receptor gamma coactivator $1 \alpha$, low density lipoprotein receptor adaptor protein-1 and STAT3 were enriched in the homeostatic process $(\mathrm{P}<0.05)$. Furthermore, the GADD45B and GADD45G were observed to interact with STAT3 and IL-6 in module one. Two types of functions of proteins in module two were identified: The starting of DNA transcription and the response to hormone stimulus. Proteins in modules three and four were predominantly enriched in hormone regulation and metabolism functions.

\section{Discussion}

Following the screening of DEGs from the gene expression profiling of the normal pituitary and pituitary adenoma tissues, differences were identified in the expression of significant genes and proteins associated with pituitary adenoma by functional enrichment analysis and PPI network construction of the DEGs. Furthermore, functional modules of protein interactions were presented with regard to the downregulated protein network. According to these results, the differential expression of genes associated with dysfunction and parasecretion of the pituitary gland, particularly the downregulation of certain transcription factor genes, significantly contributed to the occurrence of pituitary adenoma.

As the results associated with the downregulated genes demonstrated in the four functional modules, a number of proteins interacting as a whole participated in the modulation of the pituitary adenoma, the processes of which were conjoined by key transcription factors. POU1F1 is an important transcription factor found in modules three and four, the function of which is associated with hormone regulation and metabolism. POU1F1 is expressed by the anterior pituitary 


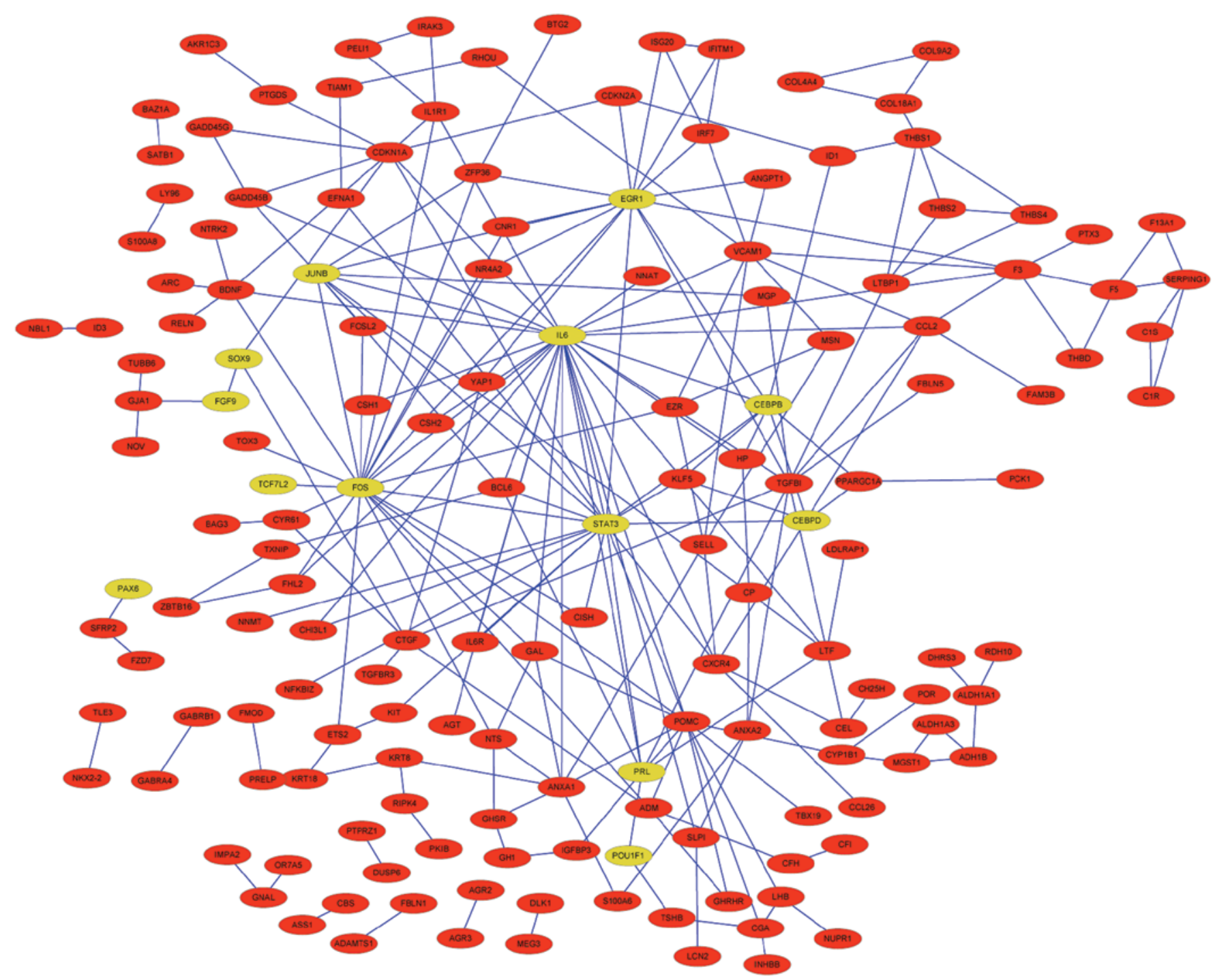

Figure 2. Protein-protein interaction networks of the downregulated differentially-expressed genes in pituitary adenoma. The 145 red nodes indicate the corresponding general proteins of the downregulated genes, while the 13 yellow nodes indicate the corresponding transcription factors.

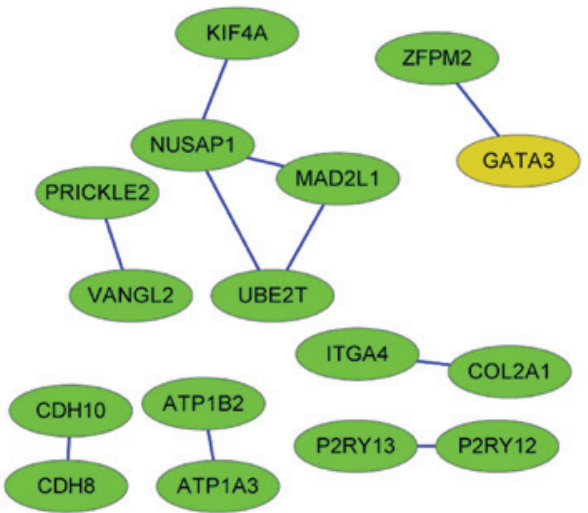

Figure 3. Protein-protein interaction network of the upregulated differentially-expressed genes in pituitary adenoma. The 15 green nodes indicate the corresponding general proteins translated for upregulated genes, while the yellow nodes indicate the corresponding transcription factors.

gland; it is important in body growth and propagation through regulating the expression of PRL, GH, Pit 1 and TSH- $\beta$ by combining with the promoters described (24). In the current study, a significant decrease of POU1F1 was detected in the pituitary adenoma tissues. This result is supported by a number of studies investigating cases of combined pituitary hormone deficiency. Carlomagno et al (25) and Turton et al (26) revealed that levels of GH, PRL and TSH decreased in the presence of diverse mutations of the POU1F1 gene. Therefore, we propose that the significantly decreased expression of the POU1F1 gene is a pivotal factor, leading to the reduction of pituitary hormone secretion in pituitary adenoma $(27,28)$. Additionally, it has been reported that two mutation sites of POU1F1 may be associated with the occurrence of combined pituitary hormone deficiency (26).

STAT3 and IL-6, which were found to be the two core nodes in module one, are pivotal transcription factors associated with immune regulation in tumors. Immune abnormalities of a number of tumors have been accompanied by the reduced expression of STAT3 and IL-6 (29). However, no direct correlation has been identified between the occurrence of pituitary adenoma and the expression of the two transcription factors.

The folliculostellate (FS) cells of the normal pituitary gland express IL-6 (30), and the secretion of IL-6 is considered to 
Table I. Significant GO terms of DEGs.

DEGs and GO terms

Downregulated genes

GO:0010033 response to organic substance

GO:0009719 response to endogenous stimulus

GO:0042127 regulation of cell proliferation

GO:0009611 response to wounding

GO:0008285 negative regulation of cell proliferation

GO:0009725 response to hormone stimulus

GO:0040008 regulation of growth

GO:0006954 inflammatory response

GO:0048545 response to steroid hormone stimulus

GO:0003006 reproductive developmental process

GO:0006952 defense response

GO:0001501 skeletal system development

GO:0045944 positive regulation of transcription

from RNA polymerase II promoter

GO:0002526 acute inflammatory response

GO:0016477 cell migration

GO:0050878 regulation of body fluid levels

GO:0006357 regulation of transcription from

RNA polymerase II promoter

GO:0030182 neuron differentiation

Upregulated genes

GO:0030001 metal ion transport

GO:0006811 ion transport

GO:0006812 cation transport
Gene

number
Bonferroni

adjusted $\mathrm{P}$-value

FDR

DEG, differentially-expressed genes; GO, gene ontology; FDR, false discovery rate.

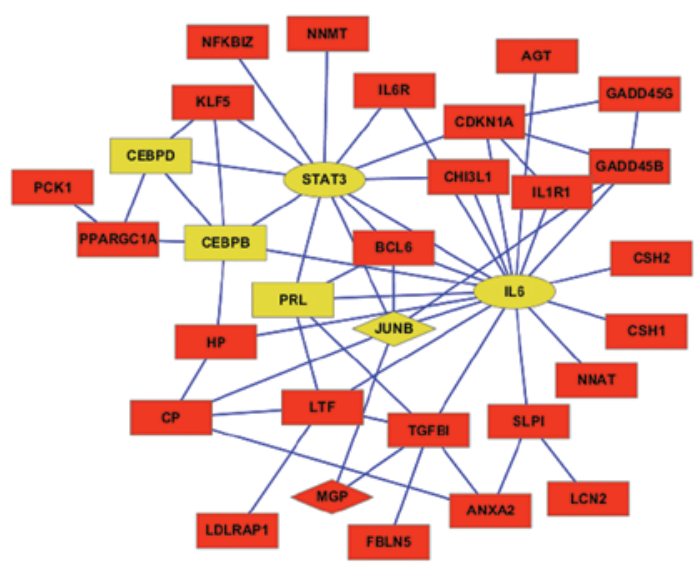

Module 1

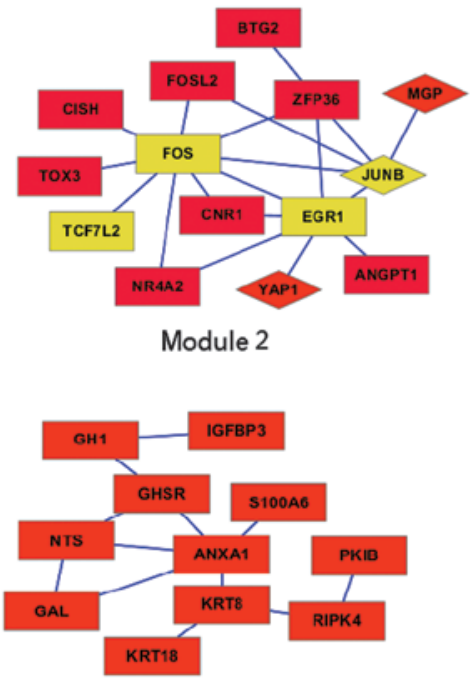

Module 4

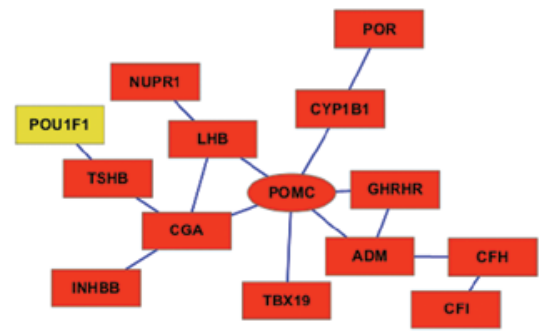

Module 3

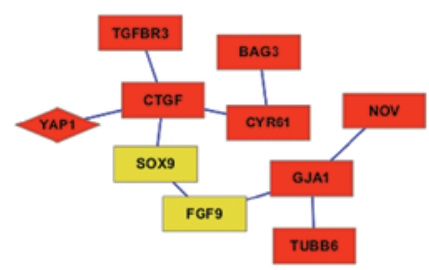

Module 5

Figure 4. Five modules from the protein-protein interaction networks of the downregulated differentially-expressed genes in pituitary adenoma. The red rectangles represent the general proteins translated by the corresponding downregulated genes in pituitary adenoma; the yellow nodes represent the transcription factors translated by the corresponding downregulated genes in pituitary adenoma. The rhombic nodes represent the proteins existing in several modules at the same time, such as JUNB, MGP and YAP1. Among the five modules, module one exhibited the largest number of nodes, the core nodes of which are the transcription factors downregulated by STAT3 and IL-6. 
Table II. Enriched functions of the five functional modules in the protein-protein interaction network of downregulated differentially-expressed genes.

\begin{tabular}{|c|c|c|c|c|}
\hline Module and GO terms & $\begin{array}{l}\text { Gene } \\
\text { count }\end{array}$ & Gene list & $\begin{array}{l}\text { Fold } \\
\text { enrichment }\end{array}$ & FDR \\
\hline \multicolumn{5}{|l|}{1} \\
\hline $\begin{array}{l}\text { GO:0070102 interleukin-6-mediated } \\
\text { signaling pathway }\end{array}$ & 3 & IL6, IL6R, STAT3 & 450.933 & 0.02057 \\
\hline GO:0042592 homeostatic process & 10 & $\begin{array}{l}\text { IL6, AGT, LTF, HP, } \\
\text { BCL6, CP, PPARGC1A, } \\
\text { LDLRAP1, STAT3, PCK1 }\end{array}$ & 6.004 & 0.02706 \\
\hline $\begin{array}{l}\text { GO:0045944 positive regulation of transcription } \\
\text { from RNA polymerase II promoter }\end{array}$ & 6 & $\begin{array}{l}\text { EGR1, FOS, NR4A2, } \\
\text { YAP1, TCF7L2, JUNB }\end{array}$ & 16.829 & 0.01404 \\
\hline GO:0009612 response to mechanical stimulus & 4 & FOS, BTG2, MGP, JUNB & 74.330 & 0.01982 \\
\hline GO:0009719 response to endogenous stimulus & 6 & $\begin{array}{l}\text { FOS, BTG2, NR4A2, } \\
\text { MGP, ANGPT1, JUNB }\end{array}$ & 15.417 & 0.02150 \\
\hline \multicolumn{5}{|l|}{2} \\
\hline GO:0010033 response to organic substance & 7 & $\begin{array}{l}\text { EGR1, FOS, BTG2, } \\
\text { NR4A2, MGP, ANGPT1, JUNB }\end{array}$ & 10.103 & 0.02166 \\
\hline $\begin{array}{l}\text { GO:0006357 regulation of transcription } \\
\text { from RNA polymerase II promoter }\end{array}$ & 7 & $\begin{array}{l}\text { EGR1, FOS, FOSL2, } \\
\text { NR4A2, YAP1, TCF7L2, JUNB }\end{array}$ & 10.020 & 0.02271 \\
\hline $\begin{array}{l}\text { GO:0045893 positive regulation } \\
\text { of transcription, DNA-dependent }\end{array}$ & 6 & $\begin{array}{l}\text { EGR } 1, \text { FOS, NR4A2 } \\
\text { YAP1, TCF7L2, JUNB }\end{array}$ & 13.090 & 0.04738 \\
\hline GO:0042445 hormone metabolic process & 5 & $\begin{array}{l}\text { CGA, CYP1B1, ADM, } \\
\text { LHB, GHRHR }\end{array}$ & 45.580 & 0.00324 \\
\hline \multicolumn{5}{|l|}{3} \\
\hline GO:0010817 regulation of hormone levels & 5 & $\begin{array}{l}\text { CGA, CYP1B 1, ADM, } \\
\text { LHB, GHRHR }\end{array}$ & 31.996 & 0.01323 \\
\hline GO:0035270 endocrine system development & 4 & $\begin{array}{l}\text { CGA, POU1F1, } \\
\text { TBX19, GHRHR }\end{array}$ & 56.017 & 0.04697 \\
\hline GO:0042445 hormone metabolic process & 5 & $\begin{array}{l}\text { CGA, CYP1B 1, ADM, } \\
\text { LHB, GHRHR }\end{array}$ & 45.580 & 0.00324 \\
\hline \multicolumn{5}{|l|}{4} \\
\hline GO:0010817 regulation of hormone levels & 5 & $\begin{array}{l}\text { CGA, CYP1B } 1, \text { ADM, } \\
\text { LHB, GHRHR }\end{array}$ & 31.996 & 0.01323 \\
\hline GO:0035270 endocrine system development & 4 & $\begin{array}{l}\text { CGA, POU1F1, TBX19 } \\
\text { GHRHR }\end{array}$ & 56.017 & 0.04697 \\
\hline
\end{tabular}

GO, gene ontology; FDR, false discovery rate.

be independent of human pituitary adenoma subtypes, which was initially determined by Jones et al (31) and confirmed by IL-6 mRNA detection $(32,33)$. The production of IL-6 in the normal pituitary gland was hypothesized to be secreted by FS cells (34); however, in pituitary adenomas, the source of IL-6 is the tumor cells (35). High serum IL-6 levels in severe cases of systemic inflammation with bacterial infections (36) indicate that IL-6 increases the cell function with regard to immunity and the inflammation response, as confirmed by experiments with IL-6-deficient mice (37).

STATs are cytoplasmic transcription factors and the key growth factors and mediators of signaling pathways and cytokines, respectively $(38,39)$. Constitutive activation of STAT3 has been observed in prostate, pancreatic, pituitary gland, brain, ovarian and a number of other cancers (40-43).
STATs normally exist in combination with the cognate receptor. In response to stimuli, phosphorylation to P-STATs occurs, followed by release from the receptor. P-STATs exert modulations to target gene expression through translocation to the nucleus (29). In the current study, it was demonstrated that the reduced expression of IL6 and STAT3 was associated with pituitary adenoma immune abnormalities. This result was consistent with the conclusion drawn by Kusaba et al (44), proposing that P-STAT3 expression is associated with carcinogenesis and tumor invasion in colorectal adenocarcinoma.

As the results in module one demonstrated, the direct combined action and coadjustment between IL-6 and STAT3 was observed on pituitary adenoma development. STAT3 is known to be activated by IL-6, and the deficiency of STAT3 often increases the accumulation of neutrophils (45). 
Furthermore, IL-6 activity is dependent on the participation of STAT3, which is significant in the transmission of IL-6 signals.

Being predominantly expressed in germinal center (GC) B-cells, BCL6 is also a downregulated protein found in module one. BCL6 is essential in pituitary adenoma due to interactions with several factors, including STAT3, IL-6 and JUNB. A study by Arguni et al (46) revealed that activated STATs leads to high BCL6 expression in GC B cells; this supports the findings in the current study, which demonstrated that low expression levels of STATs may decrease BCL6 expression and in turn, contribute to the development of pituitary adenomas.

In conclusion, the current study identified DEGs and proteins between normal pituitary gland and pituitary adenomas through establishing the gene expression profiling and PPI networks. The results of this study indicated that low expression levels of hormonal and immune-related genes facilitates the development of pituitary adenoma. The low expression of IL-6 and STAT3 was important in the dysimmunity of the pituitary adenoma. Furthermore, the low expression of POU1F1 contributed to the reduction in pituitary hormone secretion.

\section{References}

1. Woods KS, Cundall M, Turton J, et al: Over- and underdosage of SOX3 is associated with infundibular hypoplasia and hypopituitarism. Am J Hum Genet 76: 833-849, 2005.

2. Suhardja A, Kovacs K and Rutka J: Role of transcription factors in the pathogenesis of pituitary adenomas: a review. J Neurooncol 55: 185-193, 2001.

3. Burrows HL, Douglas KR, Seasholtz AF and Camper SA: Genealogy of the anterior pituitary gland: tracing a family tree. Trends Endocrinol Metab 10: 343-352, 1999.

4. Gold PW, Gwirtsman H, Avgerinos PC, et al: Abnormal hypothalamic-pituitary-adrenal function in anorexia nervosa. Pathophysiologic mechanisma in underweight and weight-corrected patients. N Engl J Med 314: 1335-1342, 1986.

5. Asa SL and Ezzat S: Genetics and proteomics of pituitary tumors. Endocrine 28: 43-47, 2005.

6. Castinetti F, Reynaud R, Saveanu A, et al: Clinical and genetic aspects of combined pituitary hormone deficiencies. Ann Endocrinol (Paris) 69: 7-17, 2008 (in French)

7. Davis SW, Castinetti F, Carvalho LR, et al: Molecular mechanisms of pituitary organogenesis: In search of novel regulatory genes. Mol Cell Endocrinol 323: 4-19, 2010.

8. Ezzat S, Asa SL, Couldwell WT, et al: The prevalence of pituitary adenomas: a systematic review. Cancer 101: 613-619, 2004.

9. Daly AF, Rixhon M, Adam C, Dempegioti A, Tichomirowa MA and Beckers A: High prevalence of pituitary adenomas: a cross-sectional study in the province of Liege, Belgium. J Clin Endocr Metab 91: 4769-4775, 2006.

10. Vogelstein B, Lane D and Levine AJ: Surfing the p53 network. Nature 408: 307-310, 2000.

11. Michaelis KA, Knox AJ, Xu M, et al: Identification of growth arrest and DNA-damage-inducible gene beta (GADD45 beta) as a novel tumor suppressor in pituitary gonadotrope tumors. Endocrinology 152: 3603-3613, 2011.

12. Sánchez-Tejada L, Sánchez-Ortiga R, Moreno-Pérez Ó, et al: Pituitary tumor transforming gene and insulin-like growth factor 1 receptor expression and immunohistochemical measurement of Ki-67 as potential prognostic markers of pituitary tumors aggressiveness. Endocrinol Nutr 60: 358-367, 2013.

13. D'Angelo D, Palmieri D, Mussnich P, et al: Altered microRNA expression profile in human pituitary $\mathrm{GH}$ adenomas: down-regulation of miRNA targeting HMGA1, HMGA2, and E2F1. J Clin Endocrinol Metab 97: E1128-E1138, 2012.

14. Yacqub-Usman K, Richardson A, Duong CV, et al: The pituitary tumour epigenome: aberrations and prospects for targeted therapy. Nat Rev Endocrinol 8: 486-494, 2012.

15. Gautier L, Cope L, Bolstad BM and Irizarry RA: affy-analysis of Affymetrix GeneChip data at the probe level. Bioinformatics 20: 307-315, 2004.
16. Durinck S, Spellman PT, Birney Eand Huber W: Mapping identifiers for the integration of genomic datasets with the R/Bioconductor package biomaRt. Nat Protoc 4: 1184-1191, 2009.

17. Dennis G Jr, Sherman BT, Hosack DA, et al: DAVID: Database for Annotation, Visualization, and Integrated Discovery. Genome Biol 4: P3, 2003.

18. Cabin RJ and Mitchell RJ: To Bonferroni or not to Bonferroni: when and how are the questions. ESA Bull 81: 246-248, 2000.

19. Jensen LJ, Kuhn M, Stark M, et al: STRING 8 - a global view on proteins and their functional interactions in 630 organisms. Nucleic Acids Res 37: D412-D416, 2009.

20. Liu ZP, Wang Y, Zhang XS and Chen L: Identifying dysfunctional crosstalk of pathways in various regions of Alzheimer's disease brains. BMC Syst Biol 4: S11, 2010.

21. Matys V, Fricke E, Geffers R, et al: TRANSFAC: transcriptional regulation, from patterns to profiles. Nucleic Acids Res 31: 374-378, 2003.

22. Smoot ME, Ono K, Ruscheinski J, Wang PL and Ideker T: Cytoscape 2.8: new features for data integration and network visualization. Bioinformatics 27: 431-432, 2011.

23. Hu Y, Chen H, Duan C, et al: Deficiency of Erbin induces resistance of cervical cancer cells to anoikis in a STAT3-dependent manner. Oncogenesis 2: e52, 2013.

24. Lan XY, Pan CY, Chen H, et al: An AluI PCR-RFLP detecting a silent allele at the goat POU1F1 locus and its association with production traits. Small Rumin Res 73: 8-12, 2007.

25. Carlomagno Y, Salerno M, Vivenza D, et al: A novel recessive splicing mutation in the POU1F1 gene causing combined pituitary hormone deficiency. J Endocrinol Invest 32: 653-658, 2009.

26. Turton JP, Strom M, Langham S, Dattani MT and Le Tissier P: Two novel mutations in the POU1F1 gene generate null alleles through different mechanisms leading to combined pituitary hormone deficiency. Clin Endocrinol (Oxf) 76: 387-393, 2012.

27. Tenenbaum-Rakover Y, Sobrier ML and Amselem S: A novel POU1F1 mutation (p.Thr168IlefsX7) associated with an early and severe form of combined pituitary hormone deficiency: functional analysis and follow-up from infancy to adulthood. Clinical Endocrinol (Oxf) 75: 214-219, 2011.

28. Reynaud R, Castinetti F, Galon-Faure N, et al: Genetic aspects of growth hormone deficiency. Arch Pediatr 18: 696-706, 2011 (In French).

29. Zhong Z, Wen Z and Darnell JE Jr: Stat3: a STAT family member activated by tyrosine phosphorylation in response to epidermal growth factor and interleukin-6. Science 264: 95-98, 1994.

30. Borg SA, Kerry KE, Royds JA, Battersby RD and Jones TH: Correlation of VEGF production with IL1 alpha and IL6 secretion by human pituitary adenoma cells. Eur J Endocrinol 152: 293-300, 2005.

31. Jones TH, Justice S, Price A and Chapman K: Interleukin-6 secreting human pituitary adenomas in vitro. J Clin Endocrinol Metab 73: 207-209, 1991.

32. Jones TH, Daniels M, James RA, et al: Production of bioactive and immunoreactive interleukin-6 (IL-6) and expression of IL-6 messenger ribonucleic acid by human pituitary adenomas. J Clin Endocrinol Metab 78: 180-187, 1994.

33. Green VL, Atkin SL, Speirs V, et al: Cytokine expression in human anterior pituitary adenomas. Clin Endocrinol (Oxf) 45: 179-185, 1996.

34. Vankelecom H, Carmeliet P, Van Damme J, Billiau A and Denef C: Production of interleukin- 6 by folliculo-stellate cells of the anterior pituitary gland in a histiotypic cell aggregate culture system. Neuroendocrinology 49: 102-106, 1989.

35. Ueta Y, Levy A, Chowdrey HS and Lightman SL: S-100 antigen-positive folliculostellate cells are not the source of IL-6 gene expression in human pituitary adenomas. J Neuroendocrinol 7 : 467-474, 1995.

36. Gaïni S, Koldkjær OG, Pedersen C and Pedersen SS: Procalcitonin, lipopolysaccharide-binding protein, interleukin-6 and C-reactive protein in community-acquired infections and sepsis: a prospective study. Crit Care 10: R53, 2006.

37. Sutherland RE, Olsen JS, Mckinstry A, Villalta SA and Wolters PJ: Mast cell IL-6 improves survival from Klebsiella pneumonia and sepsis by enhancing neutrophil killing. J Immunol 181: 5598-5605, 2008.

38. Matsukawa A, Takeda K, Kudo S, Maeda T, Kagayama M and Akira S: Aberrant inflammation and lethality to septic peritonitis in mice lacking STAT3 in macrophages and neutrophils. J Immunol 171: 6198-6205, 2003.

39. Darnell JE, Jr: STATs and gene regulation. Science 277: 1630-1635, 1997. 
40. Song JI and Grandis JR: STAT signaling in head and neck cancer. Oncogene 19: 2489-2495, 2000.

41. Scholz A, Heinze S, Detjen KM, et al: Activated signal transducer and activator of transcription 3 (STAT3) supports the malignant phenotype of human pancreatic cancer. Gastroenterology 125 : 891-905, 2003

42. Page C, Huang M, Changery J, et al: Expression of constitutive activation of AKT and Stat 3 oncogene products in ovarian, cervical, and prostate cancer cells. Proceedings of the American Association for Cancer Research Annual Meeting:728-729, 2000

43. Bowman T, Garcia R, Turkson J and Jove R: STATs in oncogenesis. Oncogene 19: 2474-2488, 2000.
44. Kusaba T, Nakayama T, Yamazumi K, et al: Expression of p-STAT3 in human colorectal adenocarcinoma and adenoma; correlation with clinicopathological factors. J Clin Pathol 58: 833-838, 2005.

45. Lee CK, Raz R, Gimeno R, et al: STAT3 is a negative regulator of granulopoiesis but is not required for G-CSF-dependent differentiation. Immunity 17: 63-72, 2002.

46. Arguni E, Arima M, Tsuruoka N, Sakamoto A, Hatano M and Tokuhisa T: JunD/AP-1 and STAT3 are the major enhancer molecules for high Bcl6 expression in germinal center B cells. Int Immunol 18: 1079-1089, 2006 